Proceedings of the 16th Czech and Slovak Conference on Magnetism, Košice, Slovakia, June 13-17, 2016

\title{
Magnetic Properties of Nanocrystalline Alloys after Electrons Irradiation
}

\author{
J. Sitek*, D. Holková, J. Dekan and P. Novák \\ Institute of Nuclear and Physical Engineering, Faculty of Electrical Engineering and Information Technology, \\ Slovak University of Technology, Ilkovičova 3, 81219 Bratislava, Slovakia

\begin{abstract}
Nanocrystalline $\left(\mathrm{Fe}_{3} \mathrm{Ni}_{1}\right)_{81} \mathrm{Nb}_{7} \mathrm{~B}_{12}$ alloy was irradiated by electron beams of doses up to $4 \mathrm{MGy}$. Irradiation had an influence on the magnetic microstructure of the studied alloys. It has manifested as a change in the mean orientation of local magnetization, intensity of the internal magnetic field, and distribution function of induction of internal magnetic field. All these parameters were determined from the measured Mössbauer spectra. The mean orientation of local magnetization was the most sensitive parameter. Structural changes were not identified by the Mössbauer spectroscopy and by X-ray diffraction. The results indicated that the changes of the microscopic magnetic parameters induced by irradiation depend on the phase composition. Intensity of internal magnetic field also contributes to resistivity of alloy against radiation.
\end{abstract}

DOI: 10.12693/APhysPolA.131.708

PACS/topics: 33.45.+x, 61.46.-w, 75.50.Kj

\section{Introduction}

In the recent years, nanocrystalline alloys have become attractive for many applications. The most prominent FINEMET, NANOPERM and HITPERM-type alloys have been frequently investigated because they exhibit excellent soft magnetic properties.

It has been already shown [1] that some physical properties of the nanocrystalline materials can be more or less affected by neutron irradiation. Changes in the orientation of the average magnetic moment were observed in neutron irradiated metallic glasses and nanocrystals [2]. The particle bombardment produces defects that may cause a realignment of magnetic domains implying a reorientation of the magnetic moments. Changes in the local neighbourhoods of the atoms affect the average hyperfine magnetic field as well as the shape of the hyperfine field distributions.

In the case of nanocrystalline alloys, which consist of crystalline nanograins embedded in an amorphous intergranular matrix an irradiation by electrons also leads to redistribution of atoms in the amorphous matrix, disturbance of regular atomic ordering of the crystal lattice, and atom exchange between the amorphous and crystalline component [3]. The mechanism of the radiation damage also depends on the constituent elements. It was shown that the most important was content of boron.

Radiation effect of different ions at different doses and energies on the structural changes of amorphous glasses was also studied [4]. Ribbons of amorphous $\mathrm{Fe}_{74} \mathrm{Cu}_{1} \mathrm{Nb}_{3} \mathrm{Si}_{16} \mathrm{~B}_{6}$ alloy were irradiated by $593 \mathrm{MeV} \mathrm{Au}$ ions to characterize the irradiation-induced structural changes.

*corresponding author; e-mail: Jozef.Sitek@stuba.sk
Our work is focused on the following structural and local magnetic modifications induced into selected alloys by irradiation by different electron doses. The method of Mössbauer spectroscopy and X-ray diffraction were used to inspect the changes in the orientation of local magnetization, in the value of the magnetic hyperfine field as well as in the volumetric fraction of the crystalline and the amorphous components of the nanocrystalline alloy after irradiation.

\section{Experimental technique}

Ribbon-shaped specimens of the master alloy were prepared by planar flow casting method. The ribbons were about $25 \mu \mathrm{m}$ thick and $10 \mathrm{~mm}$ wide. The nominal composition was $\left(\mathrm{Fe}_{3} \mathrm{Ni}_{1}\right)_{81} \mathrm{Nb}_{7} \mathrm{~B}_{12}$. Annealing was carried out in vacuum at a temperature of $540^{\circ} \mathrm{C}$ for $1 \mathrm{~h}$. Electron irradiation was performed at SMU Trenčín using a linear accelerator with a dose up to $4 \mathrm{MGy}$ with energy $5 \mathrm{MeV}$. The applied dose density of irradiation corresponds to $1.746 \times 10^{16} \mathrm{e} / \mathrm{cm}^{2}$. Simulation of electron path in the sample is shown in Fig. 1.

The Mössbauer spectra were measured by transmission geometry on a standard constant acceleration spectrometer with a source of ${ }^{57} \mathrm{Co}(\mathrm{Rh})$. All spectra were recorded at room temperature and evaluated using the CONFIT program [5], allowing simultaneous treatment of crystalline components and residual amorphous phases using individual lines and distributions of hyperfine parameters.

XRD spectra were recorded at the Diffractometer D8 Advance using Co $K_{\alpha}$ radiation. Diffraction patterns were recorded in standard geometry.

\section{Results and discussion}

The Mössbauer spectra of all samples were evaluated using a fitting model comprising two component groups, 


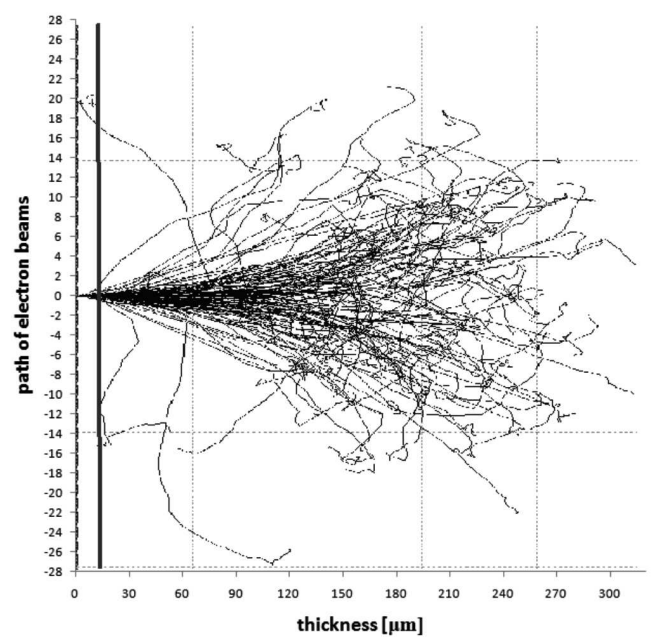

Fig. 1. Simulated path of electron beam of $1.746 \times 10^{16} \mathrm{e} / \mathrm{cm}^{2}$; thick line sign sample thickness of $25 \mu \mathrm{m}$.

i.e. the first one consists of narrow lines attributed to the Fe-atoms situated in the bulk of nanocrystalline grains and the second group describes the amorphous rest with two magnetic distributions of hyperfine magnetic field (low-field and high-field distributions). The Mössbauer spectra of the nanocrystalline sample non-irradiated and irradiated by electrons with a dose of 4 MGy recorded at room temperature are shown in Fig. 2.

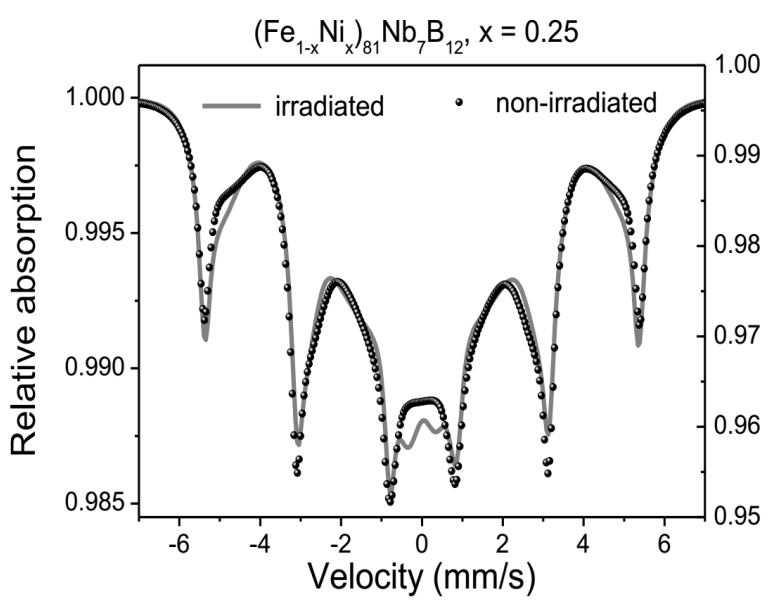

Fig. 2. The Mössbauer spectra of non-irradiated and irradiated sample.

According to the previous measurements [6, 7] performed for samples after their irradiation by heavy ions and neutrons, changes in the mean orientation of local magnetization, in the value of the average hyperfine magnetic field of the amorphous and crystalline components and of their volumetric fraction took place. Mean orientation of local magnetization is reflected by the ratio of the second (and fifth) to the third (and fourth) line intensities of a sextet in the Mössbauer spectrum $\left(A_{23}\right)$. This parameter achieves its maximum value if the mean lo- cal magnetization lays in sample surface and reaches its minimum value, when is oriented perpendicular to the sample surface. Changes in the volumetric fraction of the constituent phases were found, if a part of the crystalline component was amorphised as was found after neutron irradiation with high fluence [6]. If the structure is only modified by irradiation, the values of the internal magnetic fields and the relative amounts of the amorphous and crystalline components change usually in the frame of error. An irradiation by electrons also leads to redistribution of atoms in amorphous matrix. In our case, $A_{23}$ parameter is the most sensitive parameter, whose behavior points on some changes of the mean orientation of local magnetization.

The values of $A_{23}$ parameter obtained for investigated samples are listed in Table I. Our measurements show that mean orientation of local magnetization turns to the ribbon plane. After higher doses of electrons change of mean orientation of local magnetization is more significant in amorphous part than in crystalline one. It means that amorphous component is more sensitive to electron radiation than crystalline one. We also observed changes in inductions of internal magnetic field as is shown in Table II. Internal magnetic field of amorphous component consists of low and high field part. Their mean values and discrete values of crystalline component are given in Table II. Evaluation of distribution of induction of internal magnetic field ( $p(B)$ function) indicates some changes in magnetic structure (Fig. 3). The internal magnetic field of crystalline part only changes slightly. We also observed similar behaviour in amorphous precursor where low field part was more sensitive to radiation than high field part.

TABLE I

$A_{23}$ parameter of non-irradiated and irradiated samples.

\begin{tabular}{c|c|c|c|c}
\hline \hline \multirow{2}{*}{ Component } & \multicolumn{4}{|c}{$D$ [MGy] } \\
\cline { 2 - 5 } & - & 1 & 2 & 4 \\
\hline$A_{23}$ amorphous & 2.17 & 2.77 & 2.82 & 3.10 \\
$A_{23}$ crystalline & 3.34 & 3.34 & 3.32 & 3.44
\end{tabular}

TABLE II

Induction $B$ of internal magnetic field of non-irradiated and irradiated sample

\begin{tabular}{c|c|c|c|c}
\hline \hline \multirow{2}{*}{ Component } & \multicolumn{4}{|c}{ Induction $B[\mathrm{~T}]$ for dose of electrons } \\
\cline { 2 - 5 } & - & $1 \mathrm{MGy}$ & $2 \mathrm{MGy}$ & 4 MGy \\
\hline amorphous & 31.23 & 31.52 & 33.31 & 33.03 \\
& 13.65 & 13.40 & 13.20 & 12.85 \\
\hline crystalline & 33.40 & 33.31 & 33.40 & 33.45
\end{tabular}

Electron irradiation causes displacement of atoms from their original position. We suppose that magnetic forces contribute to interatomic forces and this is the reason that amorphous component with lower internal field is more sensitive to electron radiation than higher internal field. We suppose that in origin structure by higher doses of radiation a new crystalline phase will be created. 
In the case of crystalline component forces in crystalline structure and high internal field decrease possibility of displacement of atoms.

Our measurements did not find new phases after irradiation. This was confirmed from the measurements of the Mössbauer spectra and from X-ray diffraction measurements. XRD are shown in Fig. 4. Both main peaks correspond to iron -nickel phase.

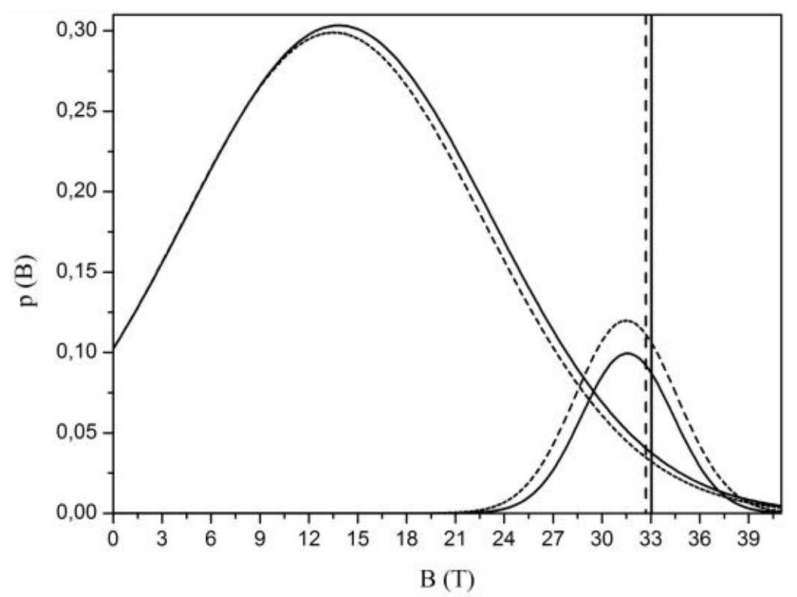

Fig. 3. $p(B)$ distribution function before (full line) and after (disconnect line) electron irradiation. Discrete lines correspond to crystalline component.

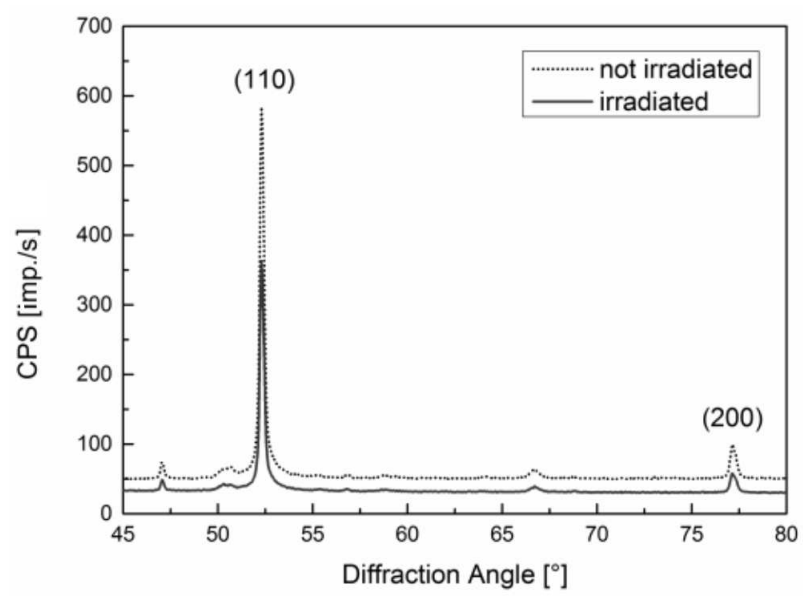

Fig. 4. XRD pattern of non-irradiated and irradiated samples.

Pattern of irradiated sample is in lower position than non-irradiated one. This phenomena is typical for these kind of samples. We suppose that in origin structure by higher doses of radiation a new crystalline phase will be created. We can mention that we observed lower pattern after irradiation also by irradiation of amorphous precursor in many samples.

\section{Conclusions}

Nanocrystalline $\left(\mathrm{Fe}_{3} \mathrm{Ni}_{1}\right)_{81} \mathrm{Nb}_{7} \mathrm{~B}_{12}$ alloys after electron irradiation of the doses up to 4 MGy were partly modified, which is indicated from mean orientation of local magnetization, values of internal magnetic field and distribution function of magnetic induction of internal magnetic field. Other Mössbauer parameters as isomer shift and quadrupole splitting changed in the frame of error. Electron irradiation causes small structural changes which reflects in parameters of magnetic structure. Components with low internal magnetic field are more sensitive to radiation than high field components. This indicates that magnetic forces could contribute to radiation resistivity. New phases were not observed. According to the previous measurements [7] new phases are created after stronger radiation damage which was achieved by neutrons. We suppose that for structural radiation damage higher doses of electrons are necessary. It also depends at the constituent elements as it was shown at FeSiBPCu nanocrystalline alloys [8], where after electron irradiation new phases were found.

\section{Acknowledgments}

This work was supported by the grant of Scientific Grant Agency of the Ministry of Education of Slovak Republic and the Slovak Academy of Sciences No. VEGA$2 / 0152 / 16$ and $1 / 0477 / 16$. The authors would like to thank for irradiation of the samples at the University Center of electron accelerator SMU in Trenčín.

\section{References}

[1] F.P. Shimansky, R. Gerling, R. Wagner, Mater. Sci. Eng. 97, 173 (1997).

[2] J. Degmova, Ph.D. Thesis, Slovak University of Technology, Bratislava 2001.

[3] T. Nagase, Y. Umakoshi, N. Sumida, Sci. Technol. Adv. Mater. 3, 119 (2002).

[4] M. Miglierini, A. Lančok, M. Pavlovič, Phys. Met. Metall. 109, 469 (2010).

[5] T. Žak, Y. Jirásková, Surf. Interf. Anal. 38, 710 (2006).

[6] J. Degmova, J. Sitek, J.M. Greneche, in: Properties and Applications of Nanocrystalline Alloys from Amorphous Precursors, Eds. B. Idzikowski, P. Svec, M. Miglierini, Kluwer Academic Publ., Dordrecht 2005.

[7] J. Sitek, J. Dekan, M. Pavlovič, Acta Phys. Pol. A 126, 84 (2014)

[8] K. Shimizu, M. Nishijima, A. Takeuchi, T. Nagase, H. Yasuda, A. Makimo, J. Japan Inst. Met. 78, 364 (2014). 